\title{
Improved of Forecasting Sea Surface Temperature based on Hybrid ARIMA and Support Vector Machines Models
}

\section{Wan Imanul Aisyah Wan Mohamad Nawia , Muhamad Safiih Lola ${ }^{a, e, *}$, Razak Zakariyab $^{b}$, Nurul Hila Zainuddinc ${ }^{c}$ Abd. Aziz K. Abd Hamid ${ }^{a}$, Elayaraja Aruchunand, Nor Shairah Azurah Nazzrol ${ }^{b}$}

a Faculty of Ocean Engineering Technology and Informatics, Universiti Malaysia Terengganu, 21030 Kuala Nerus, Terengganu, Malaysia; ${ }^{b}$ Faculty of Science and Marine Environment, Universiti Malaysia Terengganu, 21030 Kuala Nerus, Terengganu, Malaysia; ' Mathematics Department, Faculty of Science and Mathematics, Universiti Pendidikan Sultan Idris, 53900 Tanjong Malim, Perak Darul Ridzuan; ${ }^{d}$ Institute of Mathematical Sciences. Faculty of Science, University of Malaya, 50603 Kuala Lumpur, Kuala Lumpur Federal Territory, Malaysia; e Institute of Tropical Biodiversity and Sustainable Development, Universiti Malaysia Terengganu, 21030 Kuala Nerus, Terengganu, Malaysia

Abstract Forecasting is a very effortful task owing to its features which simultaneously contain linear and nonlinear patterns. The Autoregressive Integrated Moving Average (ARIMA) model has been one the most widely utilized linear model in time series forecasting. Unfortunately, the ARIMA model cannot effortlessly handle nonlinear patterns alone. Thus, Support Vector Machine (SVM) model is introduced to solve nonlinear behavior in the datasets with high variance and uncertainty. The purposes of this study are twofold. First, to propose a hybrid ARIMA models using SVM. Secondly, to test the effectiveness of the proposed hybrid model using sea surface temperature (SST) data. Our investigation is based on two well-known real datasets, i.e., SST (modis) and in-situ SST (hycom). Statistical measurement such as MAE, MAPE, MSE, and RMSE are carried out to investigate the efficacy of the proposed models as compared to the previous ARIMA and SVMs models. The empirical results reveal that the proposed models produce lesser MAE, MAPE, MSE, and RMSE values in comparison to the single ARIMA and SVMs models. In additional, ARIMA-SVM are much better than compared to the existing models since the forecasting values are closer to the actual value. Therefore, we conclude that the presented models can be used to generate superior predicting values in time series forecasting with a way higher forecast precision.

Keywords: Accuracy, Forecasting, Hybrid, Sea Surface Temperature, SVMs.

\section{Introduction}

Nowadays, precise forecast of sea surface temperature (SST) has attractive rewards and profitable advantages (Muhamad Safiih, et al., [1]). Sea surface temperature (SST) is always being associated with ARIMA model. Therefore, by developing SVM method, this will be able to provide the accurate and efficient result in any case of prediction. Zhou et al., [2] made use of multiple heterogeneous data sources to forecast data movement directions. The conclusion was made that the more active data is, the more accurate prediction could be in most combinations of data source. Weng et al., [3] evolved several expert systems to trade on stock price prediction for the upcoming day. At the end, they finalized that the addition of these online sources is convenient and handy. Akita et al. [4] used textual and numeral 
information to predict stock prices. They conclude that numerical data combined with textual data that has been translated into distributed representations using Paragraph Vector outperforms numericaldata-only techniques. Checkley et al., [5] used a microblogging website like Twitter to allow traders to express their thoughts and make predictions regarding market movement and time horizons. They found out a relationship between stock price returns and emotion, traded volume and volatility. Furthermore, the prediction time span is in minutes rather than hours or days. Shynkevich et al., [6] investigate how news reports with varied degrees of relevancy targeting target stocks might be used in tandem and appropriately weighted to improve the consequences of financial forecasts. They concluded that investors and traders may take advantages from utilizing an automated system method capable of assembling data from a variety of sources and effectively forecast changes in market prices while assisting investors and traders in their decision-making process. According to Al Wadi et al., [7] in order to achieve the most precise outcomes, a suitable model should be chosen using the Box-Jenkins approach from stock forecasting. Since the direct forecasting ignores this procedure, the result showed that it is inferior to ARIMA. Fattah et al., [8] used a time series technique to model and anticipate food market demand. Several ARIMA models are built based on the historical demand information and several statistical indicators were used to find the best fitted model. The research results have shown that ARIMA $(1,0,1)$ model exists as the best forecast model in the food production industry. Such findings give manufacturers with precise guidance for selecting the quantity of food manufacturing. Considering such a specific realization in a certain time series is formed by a linear system, according to Zhang et al. [9], is impracticable. This is because most reality cases in real-world data are way more complicated and frequently nonlinear. Burbidge et al., [10] compared SVM with various machine learning techniques which is currently used such as artificial neural network (ANN). The outcome discovered that SVM is significantly better among three tested artificial neural networks. Huang et al., [11] stated that SVM is a common learning algorithm that is distinguished by its ability to monitor decision process, the kernel function implementation, and the sparse solution. Weekly NIKKEI 225 index is used to examine the SVM's forecasting efficiency. They compared SVM with Quadratic Discriminant Analysis, Elman Backpropagation Neural and Linear Discriminant Analysis. These findings discovered SVM was able to perform better than the other two models. Alaka et al., [12] dealt with a slew of new predictive models developed with a variety of tools to predict bankruptcy. As a result of the findings, SVM was regarded as crucial tool to be chosen by researchers for the development of credit models. Kim [13] predicted test data outputs with up to $57 \%$ accuracy, far exceeding the 50 percent threshold used in recent review studies when his study initiates in examining the feasibility of appealing SVM in financial forecasting. The experimental result indicated that prediction performance may be increased if the optimum SVM parameters are chosen and came to a decision where SVM is still a very intriguing issue to research. Shin et al., [14] applied SVM to bankruptcy prediction problem. They assumed that SVM was a likely to succeed classifier for improving forecasting performance derived from small data sets for a specific purpose. Sugumaran et al., [15] described that there are several major advantages of SVM. SVM alone somehow still not able to forecast the linear basic part of load accurately despite having all those advantages. Single forecasting model will not be able to provide the accurate and efficient result since SST data are constantly uneven similar to the atmospheric temperature. Furthermore, research has shown that combining forecasts outperforms forecasting models on an individual basis. Thus, the hybrid model that combined both ARIMA and SVM model is presented. Makridakis et al. [16] discovered that combining multiple models or using a hybrid model is a common practice. This is a method for improving forecasting accuracy because the well-known M-combination of forecasts from multiple models frequently results in better forecasting performance. Pai and Lin [17] employed the ARIMA and SVM advantages to enhance a hybrid approach. Their introduced strategy does show a noticeably improvement in predicting the ARIMA and SVM capabilities of a single model. Huang and Tsai [18] presented a hybrid method for predicting stock price that uses filter-based feature selection, SOFM, and SVR. Their outcome demonstrated that using hybrid approach can reduce training time when compared to the traditional single model and able to enhance predicting precision. Ming et al., [19] measured air passenger traffic in multistep-ahead prediction using ARIMA-SVMs model. As a result, it shows both techniques approach increased the prediction accuracy for both strategies significantly. Iturriaga et al., [20] has measured the bankruptcy of U.S banks using a hybrid model. They claimed that their hybrid model outperforms current methods for predicting bankruptcy. Weng et al., [21] presented Big Databased hybrid model of garlic short-term price forecasting by using monthly average garlic price data. The 
proposed ARIMA-SVM model displayed better performance where efficiency of the hybridization of ARIMA and SVM model outperforms the single ARIMA and SVM model.

\section{Materials and methods Hybrid Model in Forecasting}

\section{Standard ARIMA}

ARIMA is a statistical method and commonly recognized as Box-Jenkins's approach. This predictive technique is used for determining the future values based on the assumption given in the preceding value. ARIMA models generally expressed as ARIMA $(p, d, q)$ (Nurul Hila et al., 2019 [22]) and can be mathematically expressed of the sea surface temperature (SST) is as follows:

$$
x_{t}=\theta_{t}+\phi_{1} x_{t-1}+\phi_{2} x_{t-2}+\cdots++\phi_{p} x_{t-p}+\varepsilon_{t}-\theta_{1} \varepsilon_{t-1}-\theta_{2} \varepsilon_{t-2}-\cdots-\theta_{q} \varepsilon_{t-q}
$$

where $x_{t}$ is the sea surface temperature (SST) obtained by differencing $d$ times, $\varepsilon_{t}$ (hypothetical white noise) is considered to have a mean of zero and to be distributed independently and identically and a constant variance of $\sigma_{\varepsilon}^{2}, p$ and $q$ are the numbers of autoregressive and moving average terms in the ARIMA model and

$$
\phi_{1}(i=1,2, \ldots p) \text { and } \theta_{1}(i=1,2, \ldots q) \text { are the model parameters that need to be predicted }
$$

Basically, this method refers to the iterative application of three steps: model identification, parameter estimation and diagnostic checking. (McKenzie, 1984 [23]; Hipel \& Mcleod, 1994 [24]; Cornillon et al., 2008 [25]; Mohd Zamri et al., 2009 [26]; Rita et al., 2013[27]; Mohd Zamri et al., 2010 [28]; Muhamad Safiih et al., 2017a [29]; Muhamad Safiih et al., 2018 [30]; Syerrina et al., 2017 [31]; Muhamad Safiih et al., 2017b [32]; Nurul Hila et al., 2019 [22]) are applied iteratively until step three does not make any improvement in the model. ARIMA has the main advantage of being able to extract the linear patterns of the datasets well and is relatively effortless to use. However, ARIMA itself is not adequate for SST forecasting since it has limitations such as often nonlinear and irregular. Therefore, we introduce the SVM model to capture nonlinear patterns existing in the SST data which might yield better result.

\section{Support Vector Machines Model}

SVMs were introduced by Vapnik[33] in 1995. This method outperforms those on the basis of the experience risk minimization principle and masterly control the higher dimension data more effectively despite a limited number of training test and possess an excellent generalization. The models pick limit support vectors from input data and thus process data quickly. The SVM regression function is written as follows:

For linear and regressive data set $\left\{x_{i}, y_{i}\right\}$ the function is formulated as follows:

$$
f(x)=w^{T} x+b
$$

The coefficient $w$ and $b$ are estimated by minimizing

$$
\frac{1}{2} w^{T} w+C \frac{1}{n} \sum_{i=1}^{n} L_{\varepsilon}\left(y_{i}, f\left(x_{i}\right)\right)
$$

where $L_{\varepsilon}$ is called the $\varepsilon$-intensive loss function and is formulated as follows: 


$$
L_{\varepsilon}(y, f(x))=\left\{\begin{array}{l}
0 \text { if }|y-f(x)| \leq \varepsilon \\
|y-f(x)| \text { others }
\end{array}\right.
$$

By introducing slack variables $\xi$ and $\xi^{*}$ that denote the length among the real values and the equaling limit value of $\varepsilon$-tube, the objective function can be written as:

$$
\min \frac{1}{2} w^{T} w+C \sum_{i=1}^{n}\left(\xi_{i}+\xi_{i}^{*}\right)
$$

subject to

$$
\begin{array}{r}
w x_{i}+b_{i}-y_{i} \leq \varepsilon+\xi_{i}^{*} \\
-w x_{i}-b_{i}+y_{i} \leq \varepsilon+\xi_{i}^{*} \\
\xi_{i}, \xi_{i}^{*} \geq 0 \\
\quad \mathrm{i}=1,2, \ldots, \mathrm{N} .
\end{array}
$$

By adding Lagrange function, difficulties in quadratic optimization can be solved where equation (5) modified to the following equation:

$$
\min \frac{1}{2} \sum_{i, j=1}^{n}\left(\alpha_{i}^{*}-\alpha_{i}\right)\left(\alpha_{j}^{*}-\alpha_{j}\right) \alpha_{i}^{T} \alpha_{j}-\sum_{i=1}^{n} \alpha_{i}^{*}\left(y_{i}-\varepsilon\right)-\alpha_{i}\left(y_{i}+\varepsilon\right)
$$

where $\alpha_{i}$ and $\alpha_{i}^{*}$ are Lagrange multipliers. The constraint is

$$
\sum_{i=1}^{n}\left(\alpha_{i}-\alpha_{i}^{*}\right)=0, \quad \alpha_{i}, \alpha_{i}^{*} \in[0, C]
$$

when a data set cannot be linearly regressed, the function in $\mathrm{Eq}(2)$ can be adjusted by mapping it to a high dimension space where the formulation is as follows:

$$
f\left(x, \alpha_{i}, \alpha_{i}^{*}\right)=\sum_{i=1}^{n}\left(\alpha_{i}-\alpha_{i}^{*}\right) k\left(x_{i}, x_{j}\right)+b
$$

Let $\mathrm{K}\left(X_{i}, X_{j}\right)=\left\{\varphi\left(X_{i}\right) \cdot \varphi\left(X_{j}\right)\right\}=\varphi^{T}\left(X_{j}\right) \varphi\left(X_{i}\right) ; \mathrm{K}(x, x)$ is the inner product of feature space also known as the kernel function. Any symmetric function that satisfies Mercer condition can be used as kernel function. In this study, the Gaussian kernel function is specified.

$$
\mathrm{K}\left(x_{i}, x_{j}\right)=\exp \left(-\left\|x_{i}-x_{i}\right\|^{2} /\left(2 \sigma^{2}\right)\right)
$$

To estimate, SVMs were used and the nonlinear behavior of the forecasting data is set as Gaussian kernels. This kernel tends to perform well under general smoothness assumptions (Pai \& Lin,2005 [17]).

\section{Hybrid model}

Because it is difficult to fully comprehend the data features in a real-world problem, hybridization of two single models can be applied where it is capable of both linear and nonlinear modelling with enhanced forecasting performance overall. Hybridizing several models can also capture various parts of the underlying patterns where it can be expressed in the following way: 


$$
y_{t}=L_{t}+N_{t}
$$

where $L_{t}$ denotes the linear component and $N_{t}$ denotes the nonlinear component of the hybrid models. These two components have to be estimated from the data. ARIMA is first fitted to the linear part and the paralleling predicted value for $\hat{L}_{t}$ is acquired. Hence, the residuals vector at time $t$-th is given by

$$
e_{t}=y_{t}-\hat{L}_{t}
$$

According to (Zhang, 2003 [34]), the residuals dataset after fitting ARIMA will contain only nonlinear relationship and can be properly represented through a linear model which in this case is SVM. The residuals are modelled by the SVMs which can be depicted in the following way:

$$
e_{t}=f\left(e_{t-1}, e_{t-2}, \ldots, e_{t-n}\right)+\varepsilon_{t}
$$

where $f$ is a nonlinear function formed by the SVMs model and $\varepsilon_{t}$ denoted as the random errors of white noise. If $\widehat{N}_{t}$ is the SVM forecasting value, then the combined forecast at time $\mathrm{t}$ is obtained as:

$$
\widehat{Y}_{t}=\hat{L}_{t}+\widehat{N}_{t}
$$

\section{Study Area}

The southern South China Sea (SCS) near Peninsular Malaysia was chosen as the study area for improvement of sea surface temperature (SST) forecasting. The southern SCS located in east coast of Peninsular Malaysia. This study only uses one station point (H2005218). In this study, two temperature datasets for $\mathrm{H} 20018$ which is SST (moderate resolution imaging spectrometer-modis) and SST (the hybrid coordinate ocean model-hycom) were used to assess the efficacy of the proposed models0000000000. H2005218's monthly temperature was recorded. As shown in Figure 1, the study location is in latitude 1.770833 and longitude 104.520828, approximately 40 kilometers from Johor land. This SST in this area were mainly influenced by monsoons system.

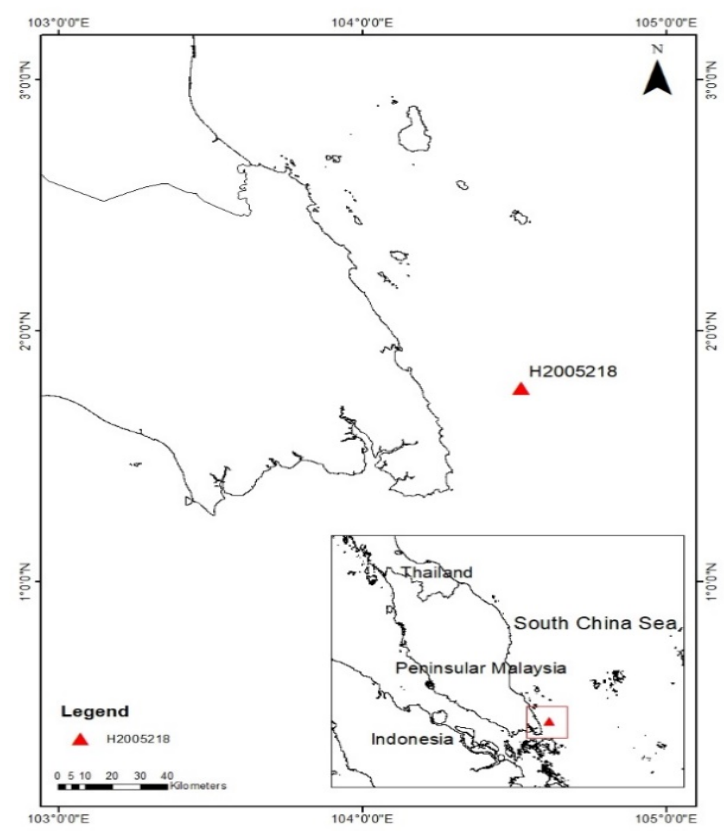

Figure 1. Map showing study area East Coast of Peninsular Malaysia 


\section{The ocean general circulation model (HYCOM)}

The Hybrid Coordinate Ocean Model (HYCOM) is produced as part of US Global Ocean Data Assimilation Experiment. HYCOM is an upgraded version of the Miami Coordinate Ocean Model (MICOM). This three-dimensional hydrodynamic ocean model improved the ocean state now casts and forecast by using the Navy Couple Ocean Data Assimilation (NCODA). NCODA is a multivariate optimum interpolation algorithm that will be used to assimilate satellite surface information, incorporating altimeter and Multi-Channel Sea Surface Temperature (MCSST) data, sea ice concentration data, and in situ profile data from XBTs (expendable bathythermographs), CTDs (conductivity temperature depth), and ARGO floats (Metzger et al., 2001[35]). Bleck, (2002[36]) in his study described HYCOM in detail and online information can be found at http://hycom.rsmas.miami.edu. The daily HYCOM SST were extracted based on time observation of MODIS overpass, respectively.

\section{Moderate Resolution Imaging Spectrometer}

MODIS is an Earth-observing instrument on board the Terra (EOS AM) and Aqua (EOS PM). MODIS Terra and Aqua were launch in December 1999 and March 2002. MODIS will shield the whole Earth's exterior within 1-2 days. Terra is scheduled to orbit the Earth from north to south over the equator in the morning, while Aqua will orbit from south to north across the equator in the midday. Each 1 to 2 days, MODIS scans the whole Earth's surface, collecting data in 36 spectral bands, wavelengths, or groups.

Satellite-derived SST were obtained from MODIS provide standard global algorithm. MODIS derived SST available at daily, weekly, and weekly. The daily and weekly level 3 MODIS SST at $4 \times 4 \mathrm{~km}$ spatial resolution data were produced by the NASA Ocean Biology Processing Group and were retrieved from the ocean color website http://modis.gsfc.nasa.gov. The data were matched up with HYCOM dataset.

\section{Sea Surface Temperature (SST)}

Sea Surface Temperature (SST) is one of the most commonly utilised factors that serves as the foundation for many oceanographic and meteorological application. SST play role of the ocean in many issues of global environment such as heat exchange, changing sea water properties and weather forecast. Wyrtki (1961) stated the water properties in SCS were influenced by the monsoons. With the advanced of satellite remote sensing and development of ocean model, SST can be observed continuously. These combinations make it possible to forecast global and regional scale of SST pattern in near-real time.

The proposed models' accuracy is calculated using statistical measures such as mean square error (MSE), the root mean square error (RMSE), mean absolute percentage error (MAPE) and the mean absolute error (MAE).

$$
\begin{aligned}
\text { MSE } & =\frac{1}{n} \sum_{j=1}^{n}\left(y_{t}-\bar{y}\right)^{2} \\
\text { RMSE } & =\sqrt{\frac{1}{n} \sum_{j=1}^{n}\left(y_{t}-\bar{y}\right)^{2}}=\sqrt{\text { MSE }} \\
\text { MAPE } & =\frac{100}{n} \sum_{j=1}^{n}\left|\frac{y_{t}-\bar{y}}{y_{t}}\right| \\
\text { MAE } & =\frac{1}{n} \sum_{j=1}^{n}\left|y_{i}-\widehat{y}_{l}\right|
\end{aligned}
$$

Where $n$ is the number of predictions, $y_{i}$ and $y_{t}$ are the real SST meanwhile $\widehat{y}_{l}, \bar{y}$ is the forecasting SST at period $t$. 


\section{Results and discussion}

\section{ARIMA Modelling}

Measurement indicators such as Akaike Information Criterion (AIC) and Bayesian Information Criterion (BIC) were used to determine the best ARIMA model. It is chosen based on the smallest AIC, BIC value that can be seen through Table 1 below:

Table 1. The best ARIMA Model selection

\begin{tabular}{lccc}
\hline Data & ARIMA $(p, d, q)$ & AIC & BIC \\
\hline SST(modis) & ARIMA $(1,0,1)$ & 159.21 & 168.15 \\
SST(hycom) & ARIMA $(0,1,0)$ & 83.4 & 85.62 \\
\hline
\end{tabular}

$\operatorname{ARIMA}(1,0,1)$ is chosen for SST(modis) meanwhile ARIMA $(0,1,0)$ is chosen for SST(hycom) as the best fitted model in this research. Since ARIMA cannot cater the nonlinear part of the dataset, SVMs play a vital role in achieving excellent forecasting performance. SVMs with Gaussian kernel functions and to determine SVM optimal parameters sets, a 10-fold cross validation procedure was applied in order to improve prediction accuracy. The parameter sets are as shown as below:

Table 2. Parameters of SVMs Model

\begin{tabular}{lcc}
\hline Data & SVM Parameter & MSE \\
\hline & $Y=1, C=8, \varepsilon=0$ & 0.3881634 \\
SST(modis) & $Y=2, C=8, \varepsilon=0$ & 0.3242707 \\
& $Y=2, C=64, \varepsilon=0.1$ & 0.3152337 \\
& $Y=2, C=256, \varepsilon=0.2$ & 0.3034296 \\
& $Y=2, C=16, \varepsilon=0.2$ & 0.3169882 \\
& $Y=2, C=32, \varepsilon=0.3$ & 0.3124604 \\
\hline SST(hycom) & $Y=2, C=256, \varepsilon=0.1$ & 0.0821869 \\
& $Y=2, C=16, \varepsilon=0.2$ & 0.1424409 \\
& $Y=2, C=128, \varepsilon=0.2$ & 0.0920611 \\
& $Y=2, C=256, \varepsilon=0.2$ & 0.0834234 \\
& $Y=2, C=64, \varepsilon=0.3$ & 0.1081246 \\
\hline
\end{tabular}

From table above, as for SST(modis) its parameters are are $y=2, C=256, \varepsilon=0.2$, meanwhile parameters for SST(hycom) are $Y=2, C=256, \varepsilon=0.1$ has the smallest error. As a result, the parameters with the lowest MSE values were chosen to be used in the best-fitting model. 

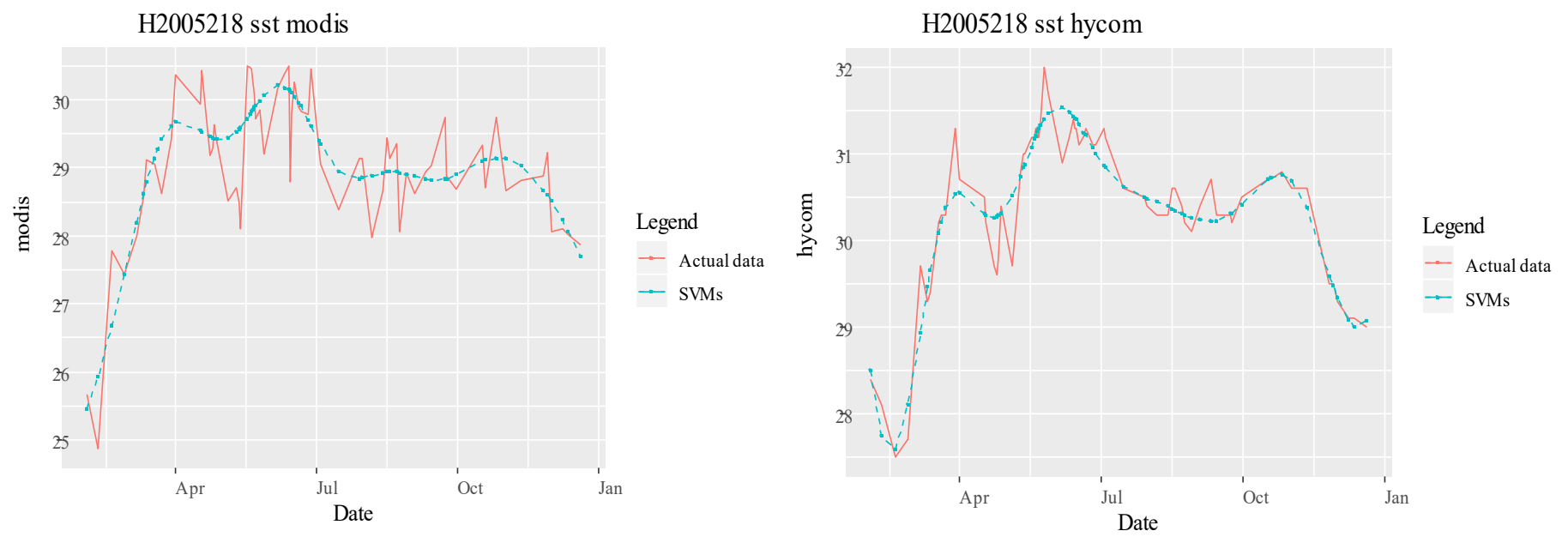

Figure 2. Points out the result of forecasting load deviation between actual data and SVMs model.

From both figures, it proves that SVMs can extract the dataset's sensitive component very well. However, neither the ARIMA nor the SVM models are capable of showing all the data patterns. Therefore, hybrid ARIMA-SVM is used as a significant reduction in overall forecasting errors.

In order to generate a smaller error term, hybrid ARIMA-SVM is employed in this section. When ARIMA error terms are fed into the SVMs in the hybrid models, final forecasting load has been obtained where can be seen in the figure below. Individual ARIMA models and SVMs models, on the other hand, are used to this forecasting load from two different SST data respectively.
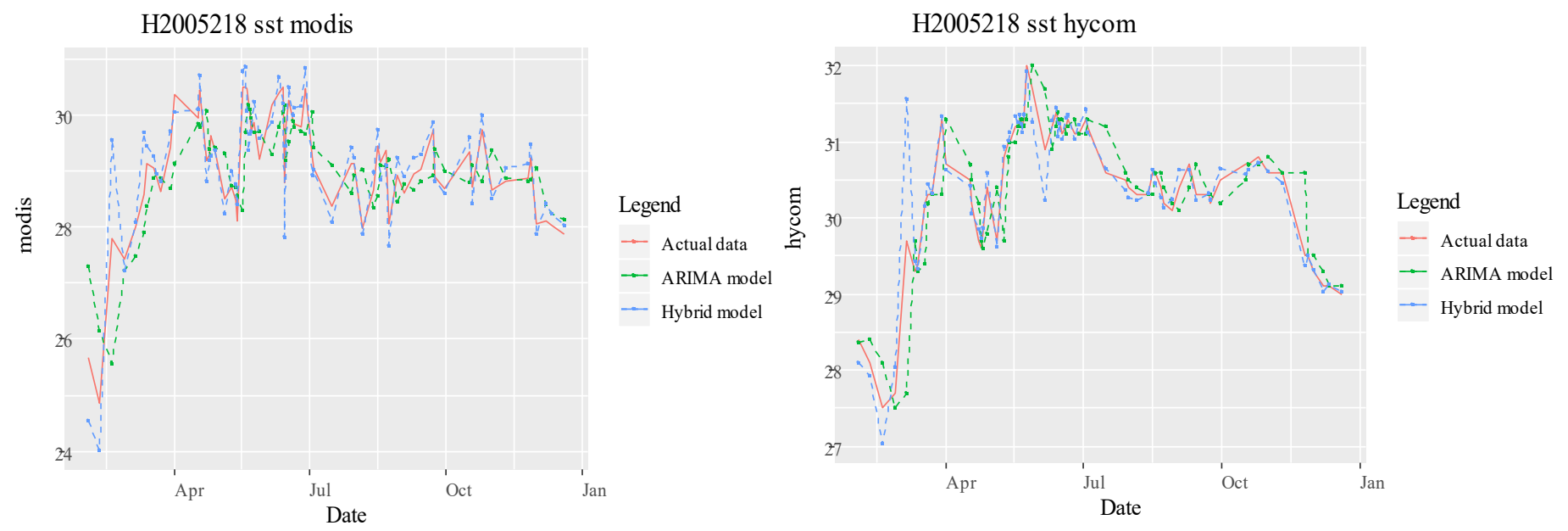

Figure 3. Compares the forecasting results from ARIMA and Hybrid models for both datasets.

It is worth noting that, despite the fact that both models exhibit the same tendency as the real data, hybrid model fits the real data better since the ARIMA model has a delay during time series. 

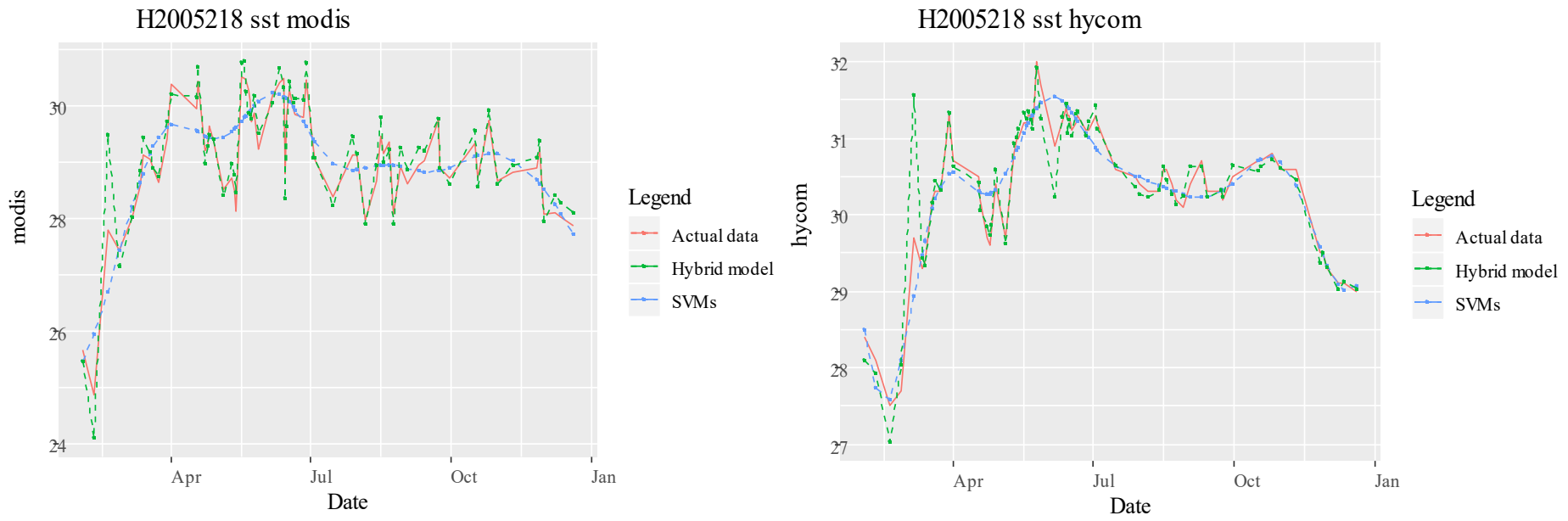

Figure 4. Compares the forecasting results from SVMs and Hybrid models for both datasets.

Similarly, to Figure 3, both models in the current figure follow the same tendency as the real data, yet the Hybrid model fits the real data better. SVMs model exhibits a delay similar in comparison to what was observed with the ARIMA model.
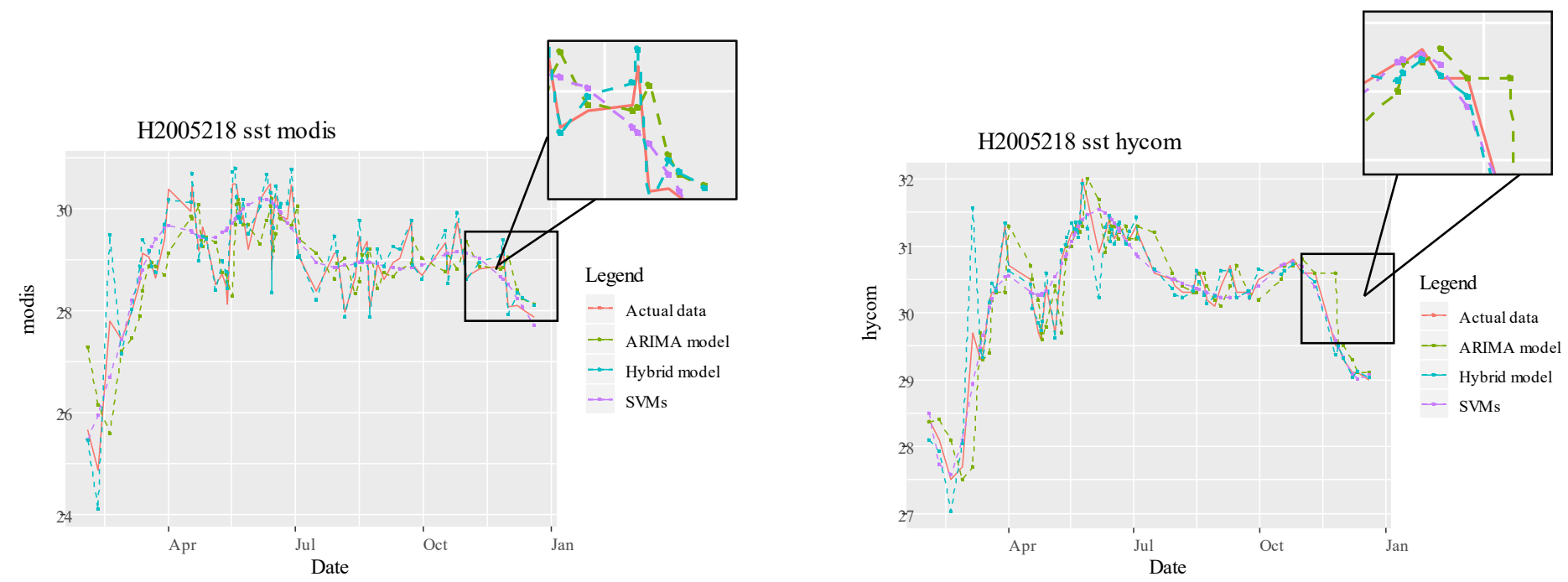

Figure 5. Predictions issued from Hybrid model fits better to the actual data than the ARIMA and SVMs working independently.

Table 3. Comparison of forecasting indices by the three different models based on SST data.

\begin{tabular}{lccccc}
\hline Data & Method & MAE & MAPE & MSE & RMSE \\
\hline SST(modis) & ARIMA & 0.5427188 & 0.018830385 & 0.5146663 & 0.7174027 \\
SST(hycom) & ARIMA & 0.2757739 & 0.009117024 & 0.19102618 & 0.4370654 \\
SST(modis) & SVMs & 0.4175986 & 0.014453146 & 0.29538648 & 0.5434947 \\
SST(hycom & SVMs & 0.1899683 & 0.006272079 & 0.07740953 & 0.2782257 \\
SST(modis) & ARIMA-SVM & 0.2261252 & 0.007861286 & 0.09781997 & 0.3127618 \\
SST(modis) & ARIMA-SVM & 0.1401983 & 0.004650276 & 0.07081886 & 0.2661181 \\
\hline
\end{tabular}


Lower MSE value indicates lower error where this error measure calculates the average squared difference between estimated value and observed value. The Hybrid model yielded significantly lower error values than those obtained with the other two models, as shown in Table 5 . This clearly demonstrates that the hybrid model is effective, accurate with higher precision in forecasting SST than the other models making its predicted values are extremely closer to actual data.

Three models are presented to estimate the behaviour of time series in two divergent SST datasets in a reasonable manner. As the outcome, it proves that ARIMA-SVM outperforms the single ARIMA along with SVMs working separately as regards estimating accurate estimation gleaned from the statistical measurement of MAE, MAPE, MSE and RMSE values. This coincides with (Pai \& Lin,2005 [17])'s result. They emphasize that combining two dissimilar models can help to lessen forecasting errors forecasting errors both theoretically and empirically. Therefore, it can be concluded that the proposed ARIMA-SVM is a superior approach for forecasting sea surface temperature where it is possible to uncover linear and nonlinear domains.

\section{Conclusions}

Since accuracy and efficiency using many statistical tools become ultimate subject in time series analysis, this study also focused on accuracy and efficiency using hybrid ARIMA-SVM to forecast the sea surface temperature (SST). The proposed model is a hybrid model that combines the nonlinear model, SVM with linear model, ARIMA and investigated its performance.

Therefore, by hybridizing, model ARIMA-SVM has been developed to predict more accurate value of real data by using two temperature datasets for H2005218 which are SST(modis) and SST(hycom) with the period of 2nd February 2009 to 20th December 2009.

For ARIMA model, after plotting the diagram it showed that the distribution of both datasets are nonstationary. To strengthen the fact, ADF test is carried out and both appeared to have greater value than alpha value which did not reject $\mathrm{H}$ null. Thus, differencing method was used and this method also helped in eliminating the seasonality. The data became stationary after first differencing. Automatic model selection criteria like AIC and BIC are utilized to lead to a firm conclusion. $\operatorname{ARIMA}(1,0,1)$ is selected for SST(modis) and ARIMA $(0,1,0)$ is chosen for SST(hycom) since they have the smallest value of AIC and BIC.

SVM model was used to deal with the nonlinear part of the dataset. To improve prediction accuracy, SVMs with Gaussian kernel functions were employed along with a 10-fold cross validation technique, to produce SVM ideal parameter values. As for SST(modis) its parameters are $\mathrm{Y}=2, \mathrm{C}=256, \varepsilon=0.2$ meanwhile parameters for SST (hycom) are $\gamma=2, C=256, \varepsilon=0.1$ has the smallest error. Therefore, the parameters with the lowest MSE values were chosen to be used in the best-fitting model.

Through statistical measurement of error estimation which are MAE, MAPE, MSE, RMSE the use of linear (ARIMA) and nonlinear (SVMs) models alone were not sufficient enough to examine the forecasting accuracy. With this evaluation, the hybrid models clearly predicted with greater precision than the ARIMA and SVM models. Furthermore, it is possible to come to a conclusion that the Hybrid model is a promising approach where both linear and nonlinear tendencies were uncovered.

\section{References}

[1] Muhamad Safiih, L, Nurul Hila, Z., Mohd Tajuddin, A., Vigneswary, P., Mohd Noor Afiq, R, Razak, Z., Md Suffian, I., and Idham,K., "Improving the Performance of ANN-ARIMA Models for Predicting Water Quality in the Offshore Area of Kuala Terengganu, Terengganu, Malaysia" Journal of Sustainability Science and Management, 13(1), 27-37, 2018.

[2] Zhou, X., Pan, Z., Hu, G., Tang, S., and Zhao, C, "Stock Market Prediction on High-Frequency Data Using Generative Adversarial Nets", Journal of Mathematical Problems in Engineering vol. 2018, 1-11, 2018. 
[3] Weng, B., Ahmed, M. A., and Megahed, F. M., "Stock market one-day ahead movement prediction using disparate data sources", Journal of Expert Systems with Applications, 79, 153-163, 2018.

[4] kita, R., Yoshihara, A., Matsubara, T., and Uehara, K., "Deep learning for stock prediction using numerical and textual", Journal of International Conference on Computer and Information Science (ICIS), pp. 2241-2891, 2016.

[5] Checkley, M. S., Higón, D. A., and Alles, H., "The hasty wisdom of the mob: How market sentiment predicts stock market behavior", Journal of Expert Systems with Applications, vol. 77, 256-263, 2017.

[6] Shynkevich, Y., McGinnity, T. M., Coleman, S. A., and Belatreche, A., "Forecasting movements of health-care stock prices based on different categories of news articles using multiple kernel learning", Journal of Decision Support Systems, vol 85, 74-83, 2016.

[7] Al Wadi S., Mohd Tahir Ismail, M. H. Alkhahazaleh, and Samsul Ariffin Addul Karim.,"Selecting Wavelet Transforms Model in Forecasting Financial Time Series Data Based on ARIMA Model", Journal of Applied Mathematical Sciences, 5(7), 315 - 326, 2011.

[8] Fattah, J., Ezzine, L., Aman, Z., El Moussami, H. \& Lachhab, A., "Forecasting of demand using ARIMA model", International Journal of Engineering Business Management 10, 184797901880867, 2018.

[9] Zhang, G., Eddy Patuwo, B., and Y. Hu, M., "Forecasting with artificial neural networks", International Journal of Forecasting, 14(1), 35-62,1998.

[10] Burbidge, R., Trotter, M., Buxton, B., and Holden, S., "Drug design by machine learning: support vector machines for pharmaceutical data analysis", Journal of Computers \& Chemistry, 26(1), 5-14, 2001.

[11] Huang, W., Nakamori, Y. \& Wang, S. Y., "Forecasting stock market movement direction with support vector machine", Computers \& Operations Research 32(10), 2513-2522, 2005.

[12] Alaka, H. A., Oyedele, L. O., Owolabi, H. A., Kumar, V., Ajayi, S. O., Akinade, O.O., and Bilal,M. "Systematic review of bankruptcy prediction models: Towards a framework for tool selection" Journal of Expert Systems with Applications, 94,164-184, 2018.

[13] Kim, K., "Financial time series forecasting using support vector machines", Journal of Neurocomputing, 55(12), 307-319. 2003.

[14] Shin, K.-S., Lee, T. S., and Kim, H. "An application of support vector machines in bankruptcy prediction model", Journal of Expert Systems with Applications, 28(1), 127-135, 2005.

[15] Sugumaran, V., Sabareesh, G., and Ramachandran, K., "Fault diagnostics of roller bearing using kernel-based neighborhood score multi-class support vector machine", Journal of Expert Systems with Applications, 34(4), 3090-3098, 2008.

[16] Makridakis, S., Andersen, A., Carbone, R., Fildes, R., Hibon, M., Lewandowski, R., and Winkler R. "The accuracy of extrapolation (time series) methods: Results of a forecasting competition", Journal of Forecasting, 1(2), 111-153, 1982.

[17] Pai, P.-F., and Lin, C.-S., "A hybrid ARIMA and support vector machines model in stock price forecasting", International Journal of Management Science, 3(3), 497-505, 2005.

[18] Huang, C.-L., and Tsai, C.-Y. "A hybrid SOFM-SVR with a filter-based feature selection for stock market forecasting", Journal Expert Systems with Applications, 36(2), 1529-1539, 2009.

[19] Ming W., Bao Y., Hu Z., and Xiong,T. Multistep-Ahead Air Passengers Traffic Prediction with Hybrid ARIMASVMs Models. The Scientific World Journal. 2014: 1-15. 2014.

[20] Iturriaga L., F. J., and Sanz, I. P., "Bankruptcy visualization and prediction using neural networks: A study of U.S. commercial banks", Journal of Expert Systems with Applications 42(6),2857-2869, 2015.

[21] Weng, B., Ahmed, M. A., and Megahed, F. M., "Stock market one-day ahead movement prediction using disparate data sources", Journal of Expert Systems with Applications, 79, 153-163, 2018.

[22] Nurul Hila, Z, Muhamad Safiih, L., Maman Abdurachman, D., Fadhilah, Y., Mohd Noor Afiq, R., Aziz, D., Yahaya, I., Mohd Tajuddin, A. Improvement of time forecasting models using a novel hybridization of bootstrap and double bootstrap artificial neural networks. Applied Soft Computing. 84, 105-676. 2019.

[23] McKenzie, E., "General exponential smoothing and the equivalent arma process", Journal of Forecasting, 3(3), 333-344, 1984.

[24] Hipel, K.W., and McLeod, A.I., "Time Series Modelling of Water Resources and Environmental Systems". Amsterdam, The Netherlands: Elsevier Science Publishers, 1994.

[25] Cornillon P., Imam W., and Matzner E., "Forecasting time series using principal component analysis with respect to instrumental variables", Journal of Computational Statistics \& Data Analysis 52(3), 1269-1280, 2008.

[26] Mohd Zamri, I., Roziah Zailan, Marzuki Ismail, and Muhamad Safiih, L., "Forecasting and Time Series Analysis of Air Pollutants in Several Area of Malaysia" American Journal of Environmental Sciences, 5(5), 625-632, 2009.

[27] Rita, S., Yony, H., Rubiyanto, Fakhri, A.M., and Madzlan, A., "Multiple Linear Regression (MLR) Modeling of Wastewater in Urban Region of Southern Malaysia", Journal of Sustainability Science and Management, 8(1), 93-102, 2013.

[28] Mohd Zamri, I., Roziah Zailan., Marzuki Ismail, and Muhamad Safiih, L., "Time-series Analysis of Pollutants in East Coast Peninsular Malaysia", Journal of Sustainability Science and Management, 5(1), 57-65, 2010.

[29] Muhamad Safiih, L., Nurul Hila, Z., Mohd Noor Afiq, R., Muhamad Na'eim, A.R, and Mohd Tajuddin A., "Improvement of Estimation Based on Small Number of Events Per Variable (EPV) using Bootstrap Logistics Regression Model. Malaysian", Journal of Fundamental and Applied Sciences, 13(4), 693-704, 2017a. 
[30] M. Z. Ibrahim, R. Zailan, M.Ismail and M.S. Lola (2010), Time-series Analysis of Pollutants in East Coast Peninsular Malaysia, Journal of Sustainability Science and Management, 5(1): 57-65.

[31] Syerrina, Z. Naeim, A.R. Muhamad Safiih L.and Nuredayu, Z., "Explorative Spatial Analysis of Coastal Community Incomes in Setiu Wetlands: Geographically Weighted Regression”, International Journal of Applied Engineering Research, 12 (18), 7392-7396, 2017.

[32] Muhamad Safiih, L, Nurul Hila, Z. Mohd Noor Afiq, R, Hizir, S., "Double Bootstrap Control Chart for Monitoring SUKUK Volatility at Bursa Malaysia", Jurnal Teknologi, 79 (6), 149-157, $2017 \mathrm{~b}$.

[33] Vapnik, V.N., "The Nature of Statistical Learning Theory", New York, Springer, 1995.

[34] Zhang, G. P., "Time series forecasting using a hybrid ARIMA and neural network model", Journal of Neurocomputing, 50,159-175, 2003.

[35] Metzger, E. J., and H. E. Hurlburt, The nondeterministic nature of Kuroshio penetration and eddy shedding in the South China Sea, J. Phys. Oceanogr, 31, 1712-1732, 2001.

[36] Bleck, R., "An oceanic general circulation model framed in hybrid isopycnic-Cartesian coordinates", Ocean Modelling, 4(1), 55-88, https://doi.org/10.1016/S1463-5003(01)00012-9, 2002. 